\title{
ESTIMACIÓN DE PARÁMETROS DE UN MODELO MATEMÁTICO EN LA TRANSMISIÓN DE VIH/SIDA EN PASTO-COLOMBIA
}

\section{PARAMETER ESTIMATION OF A MATHEMATICAL MODEL OF HIV/ AIDS TRANSMISSION IN PASTO-COLOMBIA}

\section{Marilin Nathalya Guerrero Laos ${ }^{1}$}

\section{Miller Cerón Gómez²}

\section{Eduardo lbarguen Mondragón ${ }^{3}$}

Universidad de Nariño

\section{ABSTRACT}

Since 1981, HIV has devastated a large number of lives, in which children, adolescents and adults are involved. Although death caused by HIV has been reduced thanks to "antiretroviral" drugs, it continues to spread in the population. Therefore, a mathematical model was formu1 natalyalaos@udenar.edu.co, (+57)3187327824, Universidad de Nariño, Pasto, Colombia, ORCID: 00000003-3593-6485.

$2 \quad$ millercg@udenar.edu.co, (+57)3177572322, Universidad de Nariño, Pasto, Colombia, ORCID: 0000-00022689-495X.

3 edbargun@udenar.edu.co, (+57)323 2734559, Universidad de Nariño, Pasto, Colombia, ORCID: 00000001-6308-1344. lated in ordinary differential equations to study the dynamics of HIV transmission in the City of Pasto-Colombia. The model considers the population segmented into three compartments; susceptible, infected and protected. For the analysis of the mathematical model, data from people infected with HIV during the year 2008 to 2018 were used, which served as the basis for the estimation of parameters.

\section{KEYWORDS}

HIV-AIDS, mathematical model, parameter estimation, genetic algorithm. 


\section{RESUMEN}

Desde 1981, el VIH arrasó con una gran cantidad de vidas, incluidos niños, adolescentes y adultos. A pesar de que la muerte causada por VIH se ha reducido gracias a medicamentos "antirretrovirales", este se sigue propagando en la población. Por lo anterior, se formuló un modelo matemático en ecuaciones diferenciales ordinarias para estudiar la dinámica de transmisión de VIH en la Ciudad de Pasto-Colombia. El modelo considera la población segmentada en tres compartimentos: susceptibles, infectados y protegidos. Para el análisis del modelo matemático se usaron datos de personas infectadas por VIH durante el año 2008 hasta el 2018 , los cuales sirvieron como base para la estimación de parámetros.

\section{PALABRAS CLAVE:}

VIH-sida, modelo matemático, estimación parámetros, algoritmo genético.

\section{INTRODUCCIÓN}

Se han movilizado innumerables esfuerzos para lograr el control del virus de la inmunodeficiencia humana $\mathrm{VIH}$, el cual genera preocupación en el mundo desde el punto de vista médico, político y social. Con el progreso del VIH sobre el organismo del individuo, se presenta el SIDA, el cual se caracteriza por el debilitamiento del sistema inmunológico de la persona y, por tal razón, las enfermedades oportunistas comienzan a enfermar al paciente de forma grave mediante infecciones de tipo crónico o tumores raros (Lamotte Castillo, 2014). Las rutas de transmisión son mediante vía sexual, uso de sangre o hemoderivados contaminados $y$, a través de la transmisión de la madre al feto (Uribe, Orcasita, \& Vélez, 2010). La propagación del VIH ha sido continua desde que se detectó por primera vez en 1981.

Según datos del Global Health Observatory $(\mathrm{GHO})$ de la Organización Mundial de la Salud (OMS): a finales de 2013, casi 78 millones de personas fueron infectadas con el virus del VIH y, cerca de 39 millones han muerto de VIH/SIDA, donde alrededor de 35 millones de personas vivían con $\mathrm{VIH} /$ SIDA y 1,5 millones de personas murieron de enfermedades relacionadas con el SIDA en todo el mundo. (Li, Teng, \& Miao, 2017, p. 1)

A nivel mundial, la población a quien se le debe prestar un especial cuidado son los adolescentes, debido a la conducta de riesgo en salud sexual y reproductiva que demuestran (Uribe et al., 2010), sin embargo, la epidemia de VIH en Colombia tiende a generalizarse sobre toda la población sexualmente activa (Mesa-Mazo, VergañoSalazar, Sánchez-Botero, \& Muñoz-Loaiza, 2010), por lo que se hace necesario llevar a cabo estrategias de promoción y prevención para disminuir los riesgos de infección por $\mathrm{VIH} /$ SIDA. Según el Instituto Departamental de Salud de Nariño (IDSN), la epidemia por $\mathrm{VIH} /$ SIDA en Colombia tiene una prevalencia en la población general inferior al $1 \%$, en especial, las prevalencias más altas son observadas sobre grupos de mayor vulnerabilidad, tales como hombres quienes tienen sexo con otros hombres $(\mathrm{HSH})$, personas que ejercen el trabajo sexual y usuarios de drogas endovenosas. En Colombia 2 hombres son infectados por cada mujer con VIH. El primer caso diagnosticado en el departamento de Nariño, del cual Pasto es su capital, fue en el año 1.989. En Nariño, la prevalencia es del $0.11 \%$ (aproximadamente 1 de cada 1.000 personas tiene VIH o SIDA) y, por cada 3 personas con $\mathrm{VIH} / \mathrm{SIDA}$, una es mujer. Pasto se encuentra entre uno de los municipios de Nariño con mayor prevalencia para $\mathrm{VIH}$ (IDSN, 2019).

Entender la dinámica de transmisión del $\mathrm{VIH} /$ SIDA es complicado, debido a la cantidad de variables y parámetros asociados a la enfermedad (Mesa-Mazo et al., 2010), por lo que diferentes 
autores han utilizado los modelos matemáticos para entender dicha dinámica de transmisión. Este es el caso de Rodriguez Salinas (2001) en donde se expresa que los principales modelos de transmisión de enfermedades infecciosas tales como el VIH, son los modelos SIR (Susceptibles-Infectados-Removidos), los cuales pueden ser subdivididos en modelos SI (Susceptibles-Infectados) o en el modelo de Anderson, aclarando que por ejemplo en el modelo SI, una vez el individuo contrae el VIH, este no tendrá la opción de curarse, por lo que el compartimento de personas recuperadas es una opción poco recomendable a introducir en el planteamiento de un modelo para este virus. Maimunah \& Aldila (2018)using a deterministic approach in a seven-dimensional nonlinear ordinary differential equation, we establish a mathematical model for the spread of HIV with an ART treatment intervention. In a simplified model, when no ART treatment is implemented, disease-free and the endemic equilibrium points were established analytically along with the basic reproduction number. The local stability criteria of disease-free equilibrium and the existing criteria of endemic equilibrium were analyzed. We find that endemic equilibrium exists when the basic reproduction number is larger than one. From the sensitivity analysis of the basic reproduction number of the complete model (with ART treatment plantea un modelo en EDO no lineal, formado por siete variables de estado con el cual se pretende describir lo más fielmente posible la dinámica del $\mathrm{VIH}$ y de igual manera, concibe la terapia antirretroviral (TAR) como una manera alternativa de controlar la transmisión del virus. Así mismo, Nosova \& Romanyukha (2013) desarrolla un modelo aplicado a una población conformada por individuos dependientes del alcohol y las drogas, considerando dichas dependencias factores influyentes en la propagación del VIH en Rusia . En definitiva, los sistemas de ecuaciones para modelar la dinámica de transmisión del VIH difieren principalmente en las variables, parámetros considerados y población a estudiar.
El propósito de este artículo es plantear un modelo matemático en ecuaciones diferenciales ordinarias (EDO), compuesto por los compartimentos susceptibles, infectados y protegidos (SIP), el cual debería ajustarse a la dinámica de transmisión del virus VIH en la ciudad de Pasto, Colombia. Para el planteamiento del modelo matemático SIP, se tomó como base el artículo de (Yang, 2004), el cual indica que como aún no existe una cura totalmente efectiva a una enfermedad en la cual influyen tanto aspectos biológicos como sociales, como lo es el $\mathrm{VIH} /$ SIDA, se debe llevar a cabo una protección de la población, a través de una educación preventiva y tratamiento de las personas infectadas quienes transmiten la enfermedad. El modelo planteado por Yang, se enfoca en la dinámica de transmisión del VIH entre individuos homosexuales dentro de una población, en donde se toma en cuenta para el planteamiento del sistema de EDO, los compartimentos de individuos susceptibles, latentes, infecciosos, enfermos y, protegidos. Además, los individuos infectados, se subdividen en individuos participantes y no participantes de los tratamientos disponibles para el VIH y, los individuos protegidos, son aquellos participantes de las campañas de educación sexual, los cuales se concientizan acerca del uso de preservativos, desarrollan un reconocimiento de los cofactores de la enfermedad y llevan a cabo una disminución en el número de parejas, entre otros factores.

Este artículo se estructura de la siguiente manera. En la Sección 2 se presenta un modelo epidemiológico para la dinámica de transmisión del VIH en la ciudad de Pasto-Colombia. En la sección 3 se realiza la estimación de parámetros mediante algoritmos genéticos. La sección 4 presenta una breve explicación de los algoritmos genéticos. En la sección 5 se presentan los resultados y, en la sección 6 se dan las discusiones de los resultados. 


\section{MODELO EPIDÉMICO SIP}

Para el planteamiento del modelo SIP se supone que la población total en el tiempo $t$, denotado por $N(t)$, se divide en tres compartimentos mutuamente excluyentes

- $\quad S(T)$ representa el número de personas susceptibles en el momento $t$.

- $\quad I(t)$ representa el número de personas infectadas quienes han sido diagnosticadas en el momento $t$.

- $\quad P(t)$ representa el número de personas protegidas por las campañas de educación sexual en el momento $t$.

Así, $N(t)=S(t)+I(t)+P(t)$.

Después de una unidad de tiempo, la población $S(t)$ se genera mediante una tasa de crecimiento poblacional $k$, y tiende a aumentar con la incorporación de nuevos individuos desde el compartimento de protegidos, a causa de un desinterés por las campañas de prevención con una tasa de pérdida de protección $y$. Los individuos susceptibles tienden a reducirse por el contacto efectivo con individuos infectados por $\mathrm{VIH}$ a una tasa $\beta$ y de igual manera, se reducen por el paso hacia la población protegida a una tasa $v$, la cual corresponde a un factor de protección representado por campañas de educación sexual. Gran cantidad de individuos susceptibles tienen riesgo de contraer la infección. Luego, los individuos del compartimento $I(t)$ en el tiempo $t$ se incrementan a causa de la infección de individuos susceptibles y decrece por el efecto de la enfermedad sobre el individuo, la cual genera la muerte de la persona con una tasa de mortalidad debido a la infección $\sigma$. La población del compartimento $P(t)$ aumenta por individuos provenientes directamente de $S(t)$, los cuales toman conciencia con respecto a su salud y optan por tomar en cuenta las prevenciones en contra del $\mathrm{VIH}$ con una tasa de protección $v$ y, de igual manera, el número de individuos protegidos disminuye debido al poco o nulo interés en las precauciones adecuadas para la prevención del VIH con una tasa de pérdida de protección $y$.

La definición epidemiológica de cada uno de los parámetros que intervienen en el modelo SIP se especifica en la Tabla 1.

Tabla 1. Definición epidemiológica de los parámetros considerados en el modelo SIP

\begin{tabular}{|c|c|}
\hline Notación & Parámetro \\
\hline$k$ & $\begin{array}{l}\text { Tasa de crecimiento poblacional. } \\
\text { Tasa de infección. }\end{array}$ \\
\hline$\beta$ & Tasa de pérdida de protección. \\
\hline$\gamma$ & Tasa de protección. \\
\hline$\sigma$ & $\begin{array}{l}\text { Tasa de mortalidad debida a la } \\
\text { infección por VIH. }\end{array}$ \\
\hline
\end{tabular}

Luego, la dinámica de la enfermedad por VIH/ SIDA se describe a través del siguiente modelo en EDO:

$$
\begin{gathered}
\frac{d S}{d t}=k-\beta S(t) I(t)+\gamma P(t)-v S(t) \\
\frac{d I}{d t}=\beta S(t) I(t)-\sigma I(t) \\
\frac{d P}{d t}=v S(t)-\gamma P(t)
\end{gathered}
$$

Con las siguientes condiciones iniciales:

$$
S(0)=S_{0}>0, I(t)=I_{0}>0, P(t)=P_{0}>0 .
$$

\section{ESTIMACIÓN DE PARÁMETROS}

Una concepción muy utilizada en el problema de estimación de parámetros, es la de estimar los parámetros desconocidos $p_{1}, p_{2}, \ldots p t$ de algún modelo matemático el cual describe una situación real, a través de una minimización de la distancia entre un conjunto de datos experimentales conocidos y los valores arrojados por el 
modelo teórico en ciertos puntos (Barrios Ginart, Marrero Severo, Díaz-Romañach, \& De Arazoza Rodríguez, 2010).

La estimación de parámetros en modelos de EDO con datos reales en el tiempo, por lo general suele tratarse de problemas de optimización (Barrios Ginart et al., 2010).
Para llevar a cabo la estimación de parámetros, en la Figura 1 se dispone de datos reales proporcionados por el IDSN, los cuales dan información del número de individuos infectados durante todos y cada uno de los meses comprendidos entre los años 2008 hasta 2018, es decir, se dispone del número de individuos infectados en la ciudad de Pasto en los años mencionados.

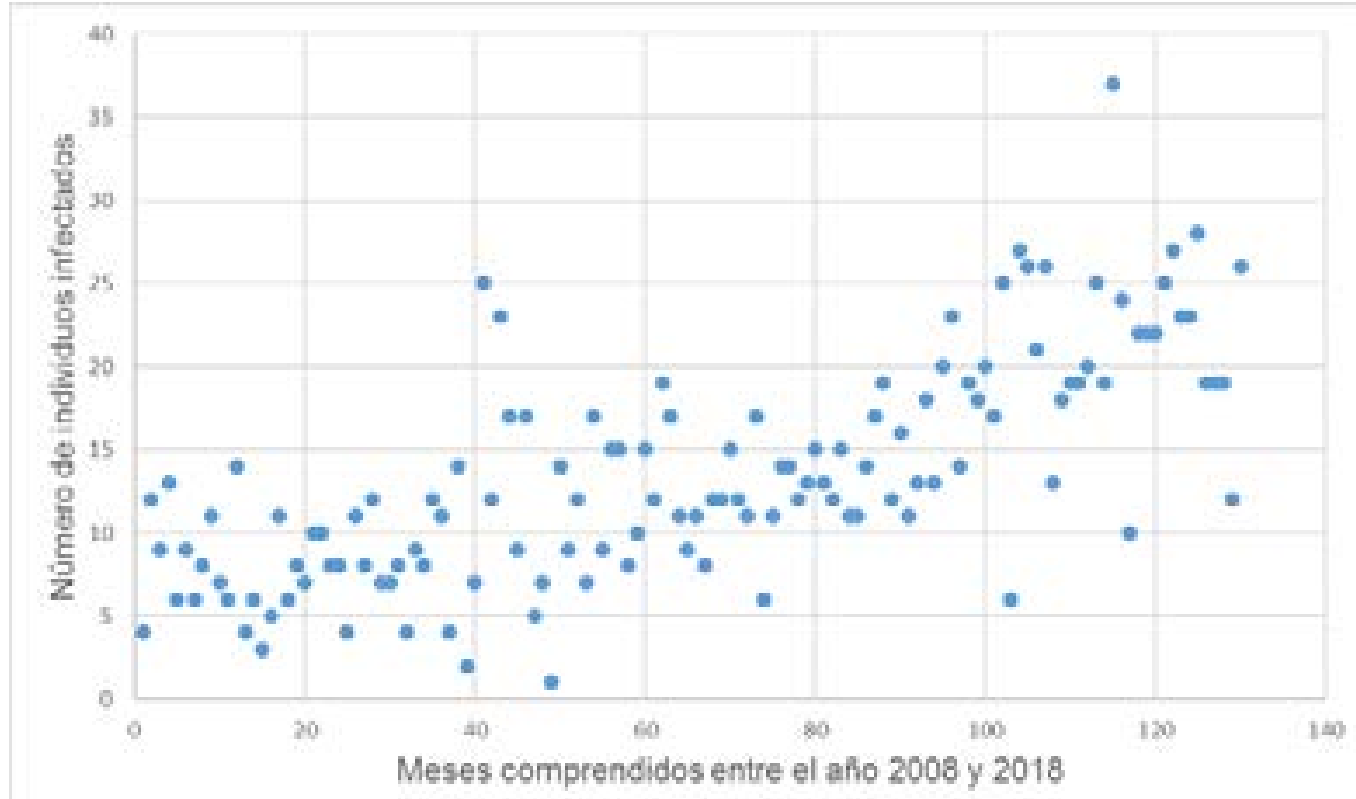

Figura 1. Datos de individuos infectados por VIH cada mes, desde el año 2008 hasta el año 2018.

Los datos fueron organizados de tal modo que describieran alguna curva ajustable, por tal razón se decidió organizarlos mediante la acu- mulación del número de individuos infectados cada mes como se muestra en la Figura 2.

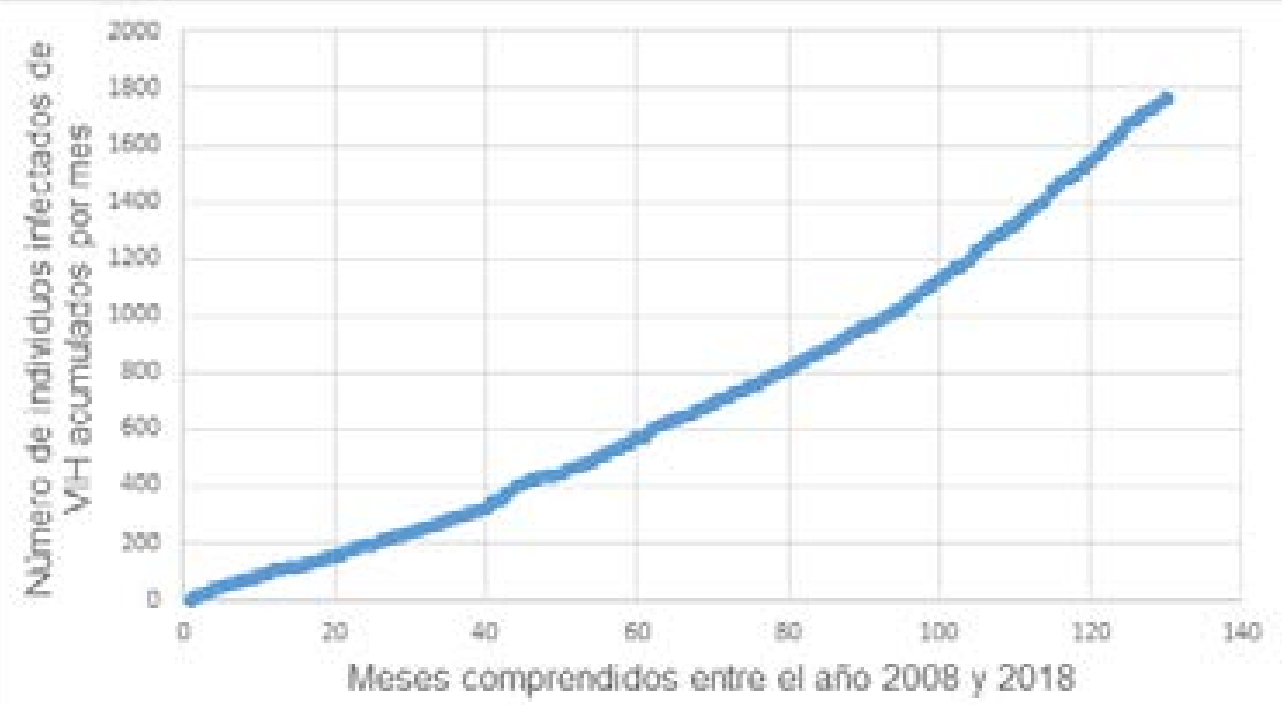

Figura 2. Datos acumulados del número de personas infectadas por VIH desde el año 2008 hasta el año 2018. 
Para llevar a cabo la estimación de parámetros del modelo SIP, se realizó simulaciones en el programa libre Octave tomando en cuenta cada uno de los elementos constituyentes de los algoritmos genéticos (AG) los cuales serán descritos en la sección 4. Con dichas simulaciones se siguió el objetivo de determinar el conjunto final de parámetros óptimos, de tal manera que contribuya significativamente al ajuste necesario de los datos observados.

\section{ALGORITMOS GENÉTICOS (AG)}

"Un algoritmo genético es una técnica de optimización que se inspira en la evolución biológica bajo la influencia de la selección natural" (Akman \& Schaefer, 2015, p. 148)

Para aplicar un AG se debe especificar el problema de optimización, el cual generalmente se lleva a cabo mediante simulaciones por ordenador. Al usar este tipo de algoritmo se tiene la ventaja de aplicar el método de optimización sin que este quede atrapado en los puntos óptimos locales. Es decir, este tipo de métodos iterativos se caracterizan por lograr la convergencia al óptimo global de la función objetivo, sin tener en cuenta restricciones tales como la complejidad o el dominio de la función (Rivas, Maldonado, Borges, \& Colmenares, 2006). En los problemas de optimización, las soluciones candidatas son etiquetadas por el término biológico "cromosomas" (Akman \& Schaefer, 2015)

Los algoritmos genéticos (AG) se enfocan en resolver problemas relacionados con encontrar buenos cromosomas y, generalmente el material de los cromosomas es manipulado sin tener previo conocimiento del tipo de problema que se está resolviendo. Para el caso de las ecuaciones diferenciales, las variables se representan como genes de un cromosoma. (Nyarko \& Scitovski, 2004)
Entonces, un AG consta de los siguientes pasos

1. Inicialice una población aleatoria de cromosomas.

2. Evalúe cada cromosoma en la función a minimizar.

3. Cree nuevos cromosomas utilizando operadores de los AG.

4. Elimine los cromosomas inadecuados de la población para dar cabida a los nuevos miembros.

5. Evalúe los nuevos cromosomas e insértelos en la población.

6. Si se cumple el criterio de detención, deténgase y devuelva el mejor cromosoma; de lo contrario, vaya al paso 3 .

En este caso, basándonos en (Gómez \& Yang, 2020) la función a minimizar es

$f(I, \delta)=\left(I-I_{\text {datos }}\right)^{2}, \quad$, donde $I$ es la solución positiva al sistema de EDO SIP, el cual representa el compartimento de personas infectadas en la población pastusa, $I_{\text {datos }}$ son los datos del número de individuos infectados en la ciudad de Pasto durante los años 2008 a 2018 y y $\delta=(\beta, \sigma, \gamma, v) \quad$ es el conjunto de los parámetros desconocidos, los cuales se desea estimar.

El primer paso es transformar cada parámetro en binario y formar una cadena llamada cromosoma. Sea lial lepresentación binaria de $\delta$.

Luego $\quad \Delta=\left(\beta_{2} \sigma_{2} \gamma_{2} v_{2}\right)$

será el cromosoma, conformado únicamente por unos y ceros $\mathrm{y}, \beta_{2}, \sigma_{2}, \gamma_{2}$ y $v_{2}$ son la representación binaria de los parámetros.

El proceso del algoritmo es el siguiente: (Gómez \& Yang, 2020) 


\section{INICIALIZACIÓN ALEATORIA DE LA POBLACIÓN DE CROMOSOMAS}

Se crea una población aleatoria de $P_{0}$ cromosomas. $\quad P_{0}=\left\{\Delta_{0}^{1}, \ldots, \Delta_{0}^{n}\right\}$

\section{EVALUACIÓN DE CADA CROMOSOMA DE LA POBLACIÓN}

En este paso procedemos a evaluar la función $f$ en cada elemento de la población $P_{0}$. Es decir, se evalúa $f\left(I, y^{i}\right)$.

\section{CREACIÓN DE NUEVOS CROMOSOMAS}

En este paso seleccionamos la siguiente población aplicando operadores genéticos (cruce y mutación)

\section{- $\quad$ Selección del método}

Para seleccionar la población, aplicamos el método de selección de torneo, que consiste en elegir aleatoriamente algún número $k$ de cromosomas y seleccionar el mínimo del conjunto $\left\{f\left(I, \gamma^{J_{1}}\right), \ldots, f\left(I, \gamma^{J_{k}}\right)\right\}$ de $k$ elementos, donde $j$ es un subconjunto de $k$ elementos $(J \subset\{1,2, \ldots, n\})$ en la próxima generación. Este proceso es repetido veces. Claramente, algunos cromosomas podrían ser seleccionados más de una vez. Ahora aplicamos los operadores de cruce y mutación a esta población seleccionada.

\section{- Operador cruce}

Para llevar a cabo el operador cruce, damos una probabilidad de cruce $p_{c}$. Esta probabilidad da el número esperado $p c x n$ de cromosomas, los cuales se someten a operación de cruce. El proceso de la función de cruce se puede visualizar en la Figura 3 y se hace de la siguiente manera: para cada cromosoma en la (nueva) población, generamos un número aleatorio $r$ en el rango $[0,1]$.

Si $r<p_{C}$ seleccionamos este cromosoma para el cruce.
Si el número de cromosomas seleccionados es par, podemos emparejar fácilmente. Si el número de cromosomas es impar, añadimos un cromosoma extra o eliminamos un cromosoma seleccionado, al azar.

Figura 3. Cruce de cromosomas.

Cromosomas seleccionados para cruce
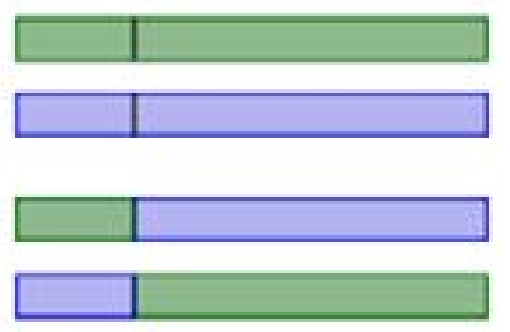

Cromosomas después de cruce

\section{- $\quad$ Operación mutación}

Este operador aplica alteraciones en los elementos de los cromosomas (cambios de 0 a 1 y viceversa) Figura 4. Damos la probabilidad de mutación $p_{m}$. Esta probabilidad nos da el número esperado de elementos mutados $p_{m} \times n \times m$.

Donde $m=\sum_{i=1}^{4} m_{i}$, con $m_{i} \quad$ comola longitud de la cadena binaria de parámetros.

El proceso para realizar el operador de mutación es similar al operador de cruce: para cada cromosoma en la población actual (es decir, después del cruce) y para cada elemento dentro del cromosoma, un número aleatorio $r$ se genera en el rango $[0,1]$. Si $r<p_{m}$, mutamos el elemento.

Figura 4. Mutación de cada elemento del cromosoma.

Cromosoma y elemento seleccionado para mutación

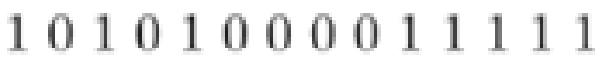

10101010011111

Cromosoma y elemento después de mutación 


\section{CRITERIO DE DETENCIÓN}

Después de todos los pasos anteriores, hemos creado la primera generación: población. Ahora, solo se repite los pasos 2 y 3 para $P_{1}$, y el proceso llega hasta las generaciones deseadas.

\section{RESULTADOS}

Las simulaciones se realizaron en el programa libre GNU Octave, versión 5.1.0 con los datos iniciales usados en las simulaciones numéricas. Ver Tabla 2.

Tabla 2. Datos iniciales usados para simulación.

\begin{tabular}{ccl}
\hline Compartimento & Población inicial & \multicolumn{1}{c}{ Información } \\
\hline Susceptibles & 405885 & $\begin{array}{l}\text { Población extraída del registro del DANE (DANE, } \\
2020) .\end{array}$ \\
Infectados & 4 & $\begin{array}{l}\text { Población extraída del Instituto departamental de } \\
\text { salud de Nariño (IDSN, 2019) } \\
\text { No se halló evidencia de individuos recibiendo orien- } \\
\text { taciones e información preventiva a través de cam- } \\
\text { pañas de educación sexual. }\end{array}$ \\
\hline
\end{tabular}

Como resultado de aplicar algoritmos genéticos al modelo SIP, con las condiciones iniciales especificadas en la Tabla 2 y, con la condición inicial de ser

$$
k=445511-405885 / 405885=0.098 \text {, }
$$

donde 445511 representa el total de la población pastusa en el año 2016 y 405885 representa el total de la población pastusa en el año 2009 , obtuvimos los datos estimados dados en la Tabla 3.

Tabla 3. Estimación de los parámetros.

\begin{tabular}{clc}
\hline Notación & Parámetro & Valor estimado \\
\hline$\beta$ & Tasa de infección. & 0.000084754 \\
$\gamma$ & Tasa de pérdida de protección. & 0.030213663 \\
$v$ & Tasa de protección. & 8.958544336 \\
$\sigma$ & Tasa de mortalidad debida a la infección & 0.096067566 \\
& por VIH. & \\
\hline
\end{tabular}


La dinámica del modelo, ajustada a los datos se

puede ver en la Figura 5

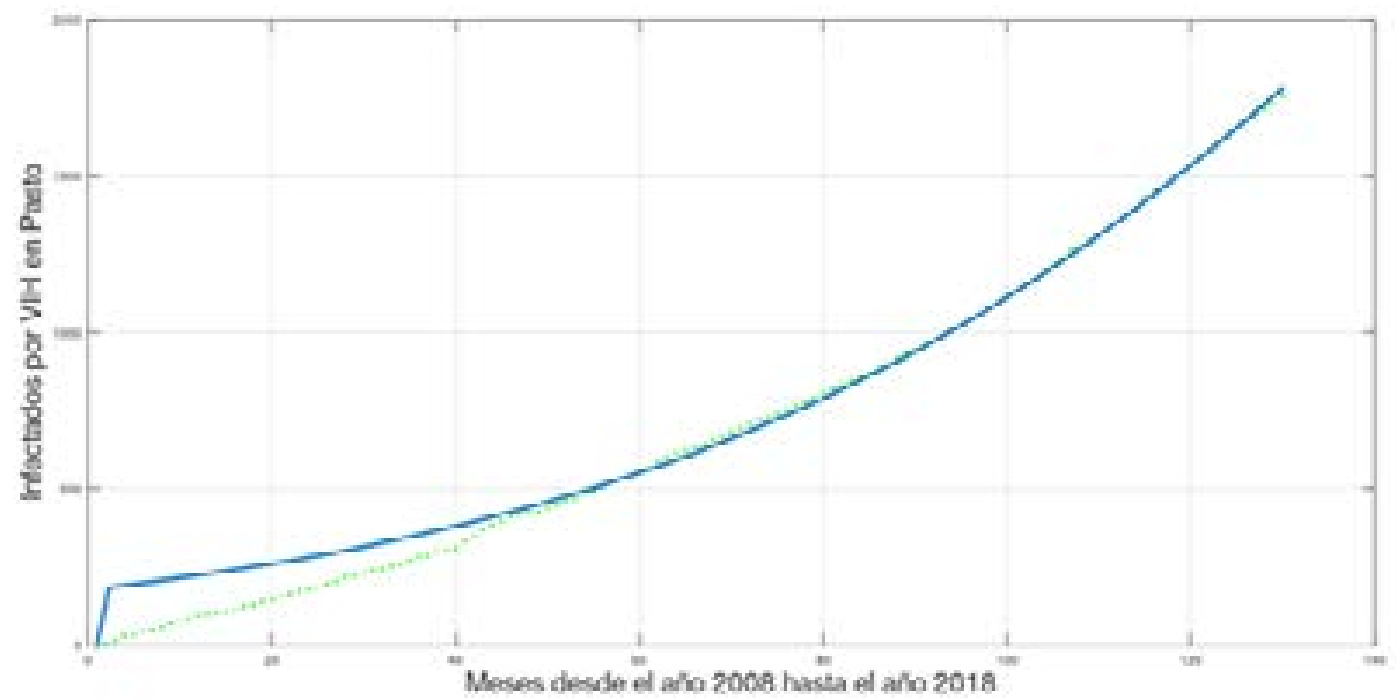

Figura 5. Ajuste de la salida del modelo con los datos experimentales acumulados por mes.

Los datos del número de individuos infectados en la ciudad de Pasto fueron tomados desde el año 2008 hasta el año 2018. Sin embargo, en la gráfica no se observa una disminución de los casos porque fueron acumulados en cada uno de los 130 meses trascurridos.

\section{REFERENCIAS BIBLIOGRAFICAS}

Akman, O., \& Schaefer, E. (2015). An evolutionary computing approach for parameter estimation investigation of a model for cholera. Journal of Biological Dynamics, 9, 147-158. https://doi.org/10.1080/1751375 8.2015 .1039608

Barrios Ginart, J., Marrero Severo, A. de los Á., Díaz-Romañach, M. L. B., \& De Arazoza Rodríguez, H. (2010). Estimación de parámetros en modelos epidemiológicos de VIH/SIDA. Revista de Matemática: Teoría y Aplicaciones, 17(2), 143. https://doi. org/10.15517/rmta.v17i2.2125
DANE. (2020). Perspectivas territoriales de desarrollo socioeconómico. Valor agregado de las estadísticas económicas y regionales.

Gómez, M. C., \& Yang, H. M. (2020). Mathematical model of the immune response to dengue virus. Journal of Applied Mathematics and Computing, 63(1-2), 455-478. https:// doi.org/10.1007/s12190-020-01325-8

IDSN. (2019). COMPARATIVO ENOS POR MUNICIPIOS - AÑOS 2009 A 2019.

Lamotte Castillo. (2014). Infección por VIH/sida en el mundo actual. Medisan, 18(7), 9931013. Retrieved from http://scielo.sld.cu/ pdf/san/v18n7/san15714.pdf

Li, Z., Teng, Z., \& Miao, H. (2017). Modeling and Control for HIVIAIDS Transmission in China Based on Data from 2004 to 2016. Computational and Mathematical Methods in Medicine, 2017, 1-13. https://doi. org/10.1155/2017/8935314 
Maimunah, \& Aldila, D. (2018). Mathematical model for HIV spreads control program with ART treatment. Journal of Physics: Conference Series, 974(1), 0-14. https:// doi.org/10.1088/1742-6596/974/1/012035

Mesa-Mazo, M. J., Vergaño-Salazar, J. G., Sánchez-Botero, C. E., \& Muñoz-Loaiza, A. (2010). Modelo matemático para la dinámica de transmisión del VIH/SIDA en una población sexualmente activa. Revista de Salud Pública, 12(2), 308-316. https://doi. org/10.1590/S0124-00642010000200014

Nosova, E. A., \& Romanyukha, A. A. (2013). Mathematical model of HIV-infection transmission and dynamics in the size of risk groups. Mathematical Models and Computer Simulations, 5(4), 379-393. https:// doi.org/10.1134/s207004821304011x

Nyarko, E. K., \& Scitovski, R. (2004). Solving the parameter identification problem of mathematical models using genetic algorithms. Applied Mathematics and Computation, 153(3), 651-658. https://doi.org/10.1016/ s0096-3003(03)00661-1

Rivas, D., Maldonado, J. L., Borges, R., \& Colmenares, G. (2006). Aplicación de los algoritmos genéticos para estimar los parámetros en un modelo de regresión de Cox. 22, 57-74.

Rodriguez Salinas, J. J. (2001). MODELOS MATEMÁTICOS DEL SIDA. Retrieved from http://eprints.uanl.mx/1042/1/1020145486. PDF

Uribe, A. F., Orcasita, L. T., \& Vélez, Y. V. (2010). Factores de riesgo para la infección por vih/sida en adolescentes y jóvenes colombianos. Acta Colombiana de Psicologia, 13(1), 11-24. Retrieved from https://repository.ucatolica.edu.co/bitstream/10983/163/1/v13n1a02.pdf
Yang, H. (2004). A population model applied to HIV transmission considering protection and treatment. Mathematical Medicine and Biology, 16(3), 237-259. https://doi. org/10.1093/imammb16.3.237 